\title{
APPROXIMATION OF BOUNDED FUNCTIONS BY CONTINUOUS FUNCTIONS
}

\author{
BY B. R. KRIPKE AND R. B. HOLMES
}

Communicated by V. Klee, June 15, 1965

We shall show that every bounded function on a paracompact space has a best approximation by continuous functions, and characterize the functions whose best approximators are unique. This is a special case of a measure-theoretic problem, whose setting is as follows. Let $X$ be a topological space and $\mu$ a Borel measure on $X$ which assigns positive mass to each nonempty open set, and has the property that $\mu(Y)=0$ if $Y$ intersects a neighborhood of each point in a $\mu$-null set. The latter condition is automatically fulfilled if each open cover of $X$ has a countable subcover. Let $L^{\infty}$ be the space of essentially bounded real-valued $\mu$-measurable functions on $X$, and give it the semi-norm $\|f\|=$ essential sup $|f|$. The bounded continuous functions on $X$ form a closed subspace $C$ of $L^{\infty}$. We say that $g \in C$ is a best approximator to $f \in L^{\infty}$ if $\|f-g\|=\operatorname{dist}(f, C)=\inf \{\|f-h\|: h \in C\}$.

If $f \in L^{\infty}$ and $x \in X, f^{*}(x)=\lim \sup _{y \rightarrow x} f(y)=\inf \{$ ess sup of $f$ over $U: U$ is a neighborhood of $x\} ; f_{*}=\lim \inf _{y \rightarrow x} f(y)$ has a similar definition. It is easy to verify that the functions $f^{*}$ and $f_{*}$ are defined everywhere, and are upper semi-continuous (usc) and lower semicontinuous (lsc) respectively.

Proposition. If $X$ is any topological space and $f \in L^{\infty}$, then $2 \operatorname{dist}(f, C) \geqq d(f) \equiv \sup \left\{f^{*}(y)-f_{*}(y): y \in X\right\}$.

PRoof. If $f^{*}(x)-f_{*}(x)>d(f)-\epsilon$ and $g \in C$ then one or the other of $\lim \sup _{y \rightarrow x}(f(y)-g(y))$ and $\lim \sup _{y \rightarrow x}(g(y)-f(y))$ is greater than $\frac{1}{2}(d(f)-\epsilon)$.

THEOREM 1. If $X$ is paracompact, then $g \in C$ is a best approximator to $f \in L^{\infty}$ if, and only if, $f^{*}-\frac{1}{2} d(f) \leqq g \leqq f_{*}+\frac{1}{2} d(f)$; every $f \in L^{\infty}$ has such $a$ best approximator; and $\operatorname{dist}(f, C)=1 / 2 d(f)$.

Proof. Since $f_{*}+\frac{1}{2} d(f) \geqq f^{*}-\frac{1}{2} d(f)$, the first pair of inequalities is equivalent to the condition that for every $\epsilon>0$ and every $x \in X$, there be a neighborhood $U$ of $x$ such that (ess sup $|f(y)-g(y)|: y \in U$ ) $\leqq \frac{1}{2} d(f)+\epsilon$. This in turn is equivalent to the assertion that for every $\epsilon>0,|f(y)-g(y)|>\frac{1}{2} d(f)+\epsilon$ only on a $\mu$-null set, which says that $\|f-g\| \leqq \frac{1}{2} d(f)$. It remains only to show that there is a continuous function which satisfies these inequalities. Since $f^{*}-\frac{1}{2} d(f)$ 
is usc and $f_{*}+\frac{1}{2} d(f)$ is lsc, this follows from the Interposition Theorem of Dieudonné [1, p. 75].

THEOREM 2. If $X$ is a normal Hausdorff space then an element $f \in L^{\infty}$ has exactly one best approximator in $C$ if, and only if, $f^{*}-f_{*}$ is a constant function.

PRoof. If $f^{*}-f_{*}$ is constant, then the function $g=f^{*}-\frac{1}{2} d(f)$ $=f_{*}+\frac{1}{2} d(f)$ is both lsc and usc, and hence is continuous. As in Theorem $1,\|f-g\|=\operatorname{dist}(f, C)$, and no other element of $C$ has this property. Conversely, we must show that if $f^{*}-f_{*}$ is not constant and $f$ has a best approximator $g$ in $C$, then it has more than one. If $f^{*}-f_{*}$ is not constant, we can choose an $\epsilon>0$ and an $x \in X$ such that $f_{*}(x)+\frac{1}{2} d(f)-\left(f^{*}(x)-\frac{1}{2} d(f)\right)=\epsilon$. Since $g$ is continuous and $f^{*}$ and $f_{*}$ are semi-continuous, there is a neighborhood $U$ of $x$ on which $|g(y)-g(x)|<\epsilon / 6, f_{*}(y)>f_{*}(x)-\epsilon / 6$ and $f^{*}(y)<f^{*}(x)+\epsilon / 6$. Since $\{x\}$ is closed and $X$ is normal, Urysohn's Lemma asserts the existence of a non-negative function $p \in C$ such that $\|p\|=\epsilon / 6$ and that $p$ vanishes outside $U$. One or the other of the inequalities $f_{*}(x)+\frac{1}{2} d(f)$ $-\epsilon / 2 \geqq g(x)$ and $g(x) \geqq f^{*}(x)-\frac{1}{2} d(f)+\epsilon / 2$ must hold, so that either $f_{*}(y)+\frac{1}{2} d(f)-\epsilon / 6>g(y)$ or $g(y)>f^{*}(y)-\frac{1}{2} d(f)+\epsilon / 6$ on $U$. According to which is the case, put $h=g+p$ so that $g \leqq h \leqq f_{*}+\frac{1}{2} d(f)$ on $U$, or $h=g-p$ so that $f^{*}-\frac{1}{2} d(f) \leqq h \leqq g$ on $U ; h=g$ on the complement of $U$. Then $h$ is also a best approximator to $f$ out of $C$.

If $\mu$ is the measure which assigns mass 1 to every point in $X$, then it certainly assigns positive mass to each nonempty open set, and mass 0 to each set which intersects a neighborhood of every point in a set of measure 0 . In this case, $L^{\infty}$ is the Banach space of all bounded functions on $X$, and \|\| is the supremum norm. Theorems 1 and 2 thus solve, as a special case, the problem of approximating bounded functions by continuous functions in the uniform norm.

\section{REFERENCE}

1. J. Dieudonné, Une généralisation des espaces compacts, J. Math. Pures Appl. 23 (1944), 65-76.

University of California, Berkeley and

Wright-Patterson Air Force Base, Ohio 